\title{
Effective Remote Monitoring System for Heart Disease Patients
}

\author{
Khalid BinSalman \\ eHealth Deveelopment \\ Security Forces Hospital \\ Riyadh, KSA \\ khalidsalman@hotmail.com
}

\author{
Amjad Fayoumi \\ Information systems Group \\ Management Science, LUMS \\ Lancaster University, UK \\ a.fayoumi@lancaster.ac
}

\begin{abstract}
Despite the advancement that has been seen in all life aspects and in particular in technology, patients still struggle in receiving the care and emergent support they need due to the ever-increasing cost of healthcare services and the increasing number of chronic diseases patients. Information technology can offer promising solutions to 21 'st century human, in particularly the what is called internet of things (IoTs) and remote based services. We design and develop a solution where patients can use wearable sensors that can offer a prediction and alerting in their heart disease conditions. The solution seems promising when it is combined with medical profile data, better decisions can be made and alerting of emergency can be timely which can help to save lives. We use data gathered from few number of people to build our analytics and decision model.
\end{abstract}

Keywords-Internet of Things, Sensors, Heart diseases, Healthcare monitoring

\section{INTRODUCTION}

Sensors can be defined as a small device that used to detect certain measurements in the environment [1]. Today, sensors became part of our lives, and sometimes we use these sensors without being aware or even thinking about how they work. For example, smartphones, smartwatches, ambient systems and what is called the internet of things (IoTs) became industry and life substantial. Many of the new mobile applications are interlinked or integrated with different type sensors e.g. accelerometer sensor, thermometer sensor, heart rate monitor sensors, fingerprint sensor, geospatial and others [2]. Equally many of the white goods now are connected to different type of sensors and to the internet. The benefit of these sensors lie in the data that is collected, we become more relying on information that are made ready to us through the information communication technologies (ICT). Sensors as a sub-class of ICT can be utilized in a way that helps in protecting many people's lives or improve their lifestyle. One of the segments of patients that can benefit from this is chronic heart disease patients. There is a risk of death threaten those patients with such disease and their heart functionality may stop at any time as consequences of this illness. Globally, the number one that causes death is cardiovascular according to [3]. However, Road and Oswego [4] report that the mortality rate of heart disease patients can be reduced to the half by adopting remote monitoring healthcare systems. Monitoring heart health requires monitoring two important measures: a) heartbeat rate and b) blood pressure rate. Normally, these two measures go up and down simultaneously. If one of these measures, for example, increased and the other one remains at the same level or decreased, then that means there is an issue. Furthermore, each measure has a normal range, and if the collected measurements are not within the healthy range, then that indicates a problem as well [5]. For instance, heartbeat rate ranges from (60) to (100) for adults [6]. While the blood pressure normal rate is (80/120) [7]. Hence, in order to remotely monitor chronic heart disease patients, wearable sensors and data analytics mechanism can be utilized to provide an effective and continuous monitoring system for those patients, by considering heartbeat rate and breath rate. With taking into consideration the difference of normal range of each patients' segments.

This paper aims to effectively utilize sensing technology to monitor heart disease patient remotely. Having said that, such as system will help reducing death rate of heart disease patient to the half if used continuously. Not only patient will benefit from this system, but also physicians and healthcare providers will be able to offer an affective remote monitoring system for patients in their homes. The system consists of three components: Sensor, Desktop Application (Client) and Web Based System (Server System). The sensor captures the vital signs data from patients and transfer it to the client application. The client application in its turn collects captured data, does the data sampling, and stores the data in a cloud-based database of the system that can be accessed anytime remotely.

\section{LITERATURE REVIEW}

\section{A. Sensor Utilization in Monitoring Patient Remotely}

Since 2003, there are a significant number of research have been conducted in respect to the utilization of wearable sensors to monitor patients. Researchers predict that these sensors and technologies might change the way we adopt and deliver healthcare. One of these research is done by Gay and Leijdekkers [8] to provide a personal heart monitoring service for heart disease patients. A wireless sensors and smart phone were used to achieve the goal of this solution. 
The electrocardiogram (ECG) data was collected and analyzed on a real-time, then according to the analyzed data, the smart phone can determine whether the patient need support or not. If the patient needs support, the caregiver will be notified and an ambulance will be called. The collected data is pushed to a healthcare data base whenever the internet connection is available. Also, GPS coordinates are taken as well to be able to allocate the patient's location in cases of emergency. The authors have demonstrated the application in a case study as a proof of concept. However, this system uses a lot of different sensors, each sensor has its own configuration and settings, which might cause a lot of hassles and discomfort to the patients and they might not be able to tolerate this kind of complexity and eventually, they might abandon using the system. A review-based research discusses a wide number of healthcare technologies has highlighted the critical value of these technologies. The review listed number of wearable technologies such as sensors, Wi-Fi, linked devices, tele-monitoring and tracking [9]. The result shows high reliability and effectiveness of preventive procedures in many areas in healthcare. It also shows that the usefulness and accuracy of most of the sensors in medical operations as well as in the practice of rehabilitation [9].

Another research examines the capabilities of sensor use conducted by Chan et al [10], the research is an experimental study using sensors where they divided the participants into groups. Each group was asked to perform certain activities. During the activities, the sensor collected and transmitted the measurements. These measurements include heartbeat rate, body temperature, physical activities of the patient, respiration rate and more. The collected data sent across Wi-Fi network to a smart phone where it gets stored and processed. Part of the data is pushed to a cloud based server for further analysis and to notify the caregiver if there is a dangerous situation. The study shows that patients can be monitored remotely with a multifunction small sensor with a high level of precision in reading compare with traditional devices. This sensor is developed by VitalConnect and is called HealthPatch MD. Thus, there was no explicit explanation of how the data get processed. In addition, the essence of data that pushed to the cloud server is not clearly mentioned and not justified. Also, the mechanism used in notifying caregivers is not explained.

While Selvaraj [11] conducted a case study based research that aims to assess HealthPatch wireless sensors for a long period of time, to monitor patients remotely. The participant had to wear the sensors to be able to measure the vital sign. The measurements include respiratory rate, heartbeat rate, and body temperature. During the first four days, the performance of the sensors was assessed and they show outstanding performance. In addition, $88.2 \%$ of the patients were comfortable with the wearable sensors. The result of this experiment shows that HealthPatch sensor can be worn comfortably, and it can be efficient and reliable in terms of remote health monitoring. Indeed, this research shows that patients can set at home and still can be monitored for a long period of time. The thing that concerns me the most is the patient experience. The result shows that $88 \%$ of the patients were convenient of wearing the sensors, which, we think, a promising result for a manufacturing company. Because, normally, attaching a device to a human body cause discomfort in most cases. So, it obvious that, technologies need to be heavily involved in order to develop a comfy and lightweight wearable healthcare system. A research conducted by Burrows et al. [12], shows how people deal with similar household technologies and assess their behavior. It suggests to engineers to consider 15 characteristics in order to design an effective and usable home-based healthcare system. These characteristics built around four subjects, a) Household technologies, b) personal experience of these technologies, c) healthcare experience and the d) thought of the smart home from user perspective. Therefore, as this research indicates, user experience is very important to build a flexible and usable healthcare system. Unlike the other researches, this research focuses on the usability and the flexibility of the delivered system.

Moreover, Bloss [13] has conducted a research aims to provide an assessment of the latest technologies in relation to wearable sensors that used for the monitoring purpose in different industries including the medical. Based on a secondary review, he offers an insight into the efforts that have been done in both academia and industry alike in terms of utilizing sensing technology for monitoring. It briefly demonstrates a number of applications of sensors such as heart attack prediction and heartbeat monitoring. One of these the project that stated briefly is Simband, which is a project that has been achieved at the University of California collaboratively with Samsung. In the mentioned project blood pressure, heartbeat rate and ESC data is collected and analyzed to predict heart attacks before it happened. On the other hands, this paper brings a new idea which is continuous patients mentoring might replaces laboratory tests. This raises a question, are we ready in terms of technology for this idea? Liu et al [14] have conducted a research tried to answer this question. The aim of Liu's research is to assess the different technologies that used for patient monitoring and to assess the maturity level of smart-homes. The result of this research shows that the readiness of this kind of technologies and this type of house is low, which means that this technology and this type of home are not ready yet.

Nevertheless, another research conducted by Alshurafa et al [15] shows a positive result of adopting systems for monitoring patients' health remotely. The aim of this is to provide better healthcare for a group of women with particular ethnicity remotely. The age of those women range from 25 to 45 . They are coached to follow a certain lifestyle to avoid known risk factors of cardiovascular. A smartphone based system is used to educate participants to follow this kind of lifestyle, they are supported based on the feedback. Then a framework is developed to recognize the classification scheme which helps to predict successful and unsuccessful subjects. The characteristics of both successful 
and unsuccessful baselines are analyzed, and the data of blood pressure, participant response and other activities are collected for the first month. The prediction tool allows researchers in clinical and science field to identify who is going to benefit the most from the remote health monitoring system. As a result, money and resources can be saved. This study shows that a number of patients have benefited from remote monitoring health. Which gives an indication that patients are ready to utilize this kind of technologies and systems when provided.

To conclude, the previous researches have made considerable progress on healthcare technologies in different directions. Despite the current capabilities we believe there is a number of issues yet to be addressed:

It is clear that there is a gap in using machine learning and data analytics of heart disease monitoring systems. Making sense of the gathered data might increase the efficiency of remote monitoring systems.

- User experience should also be taken into account. It is very important to understand the perception of users on the current wearable technologies and how they conceive its usefulness

Therefore, we aim at building a remote healthcare system for chronic heart disease, with data analytics capability, then we aim to extend the study to understand specialists perception on such as technologies.

\section{METHODOLOGY}

The applied methodology to this project is based on the methodology proposed by Peffers et al. [16] the design science research methodology for information systems research. This methodology can be applied through six phases: "Identifying problem and motivation, defining objectives, design and development, demonstration, evaluation and communication". The following sections explain how these steps were used to conduct this research. Identifying the problem done by conducting a literature review and conducting the literature critique. Then, problem can be identified and values become clearer. There were demands for utilizing technology in order to provide remote monitoring system. Nonetheless, the provided solutions did not provide the value that satisfy patients' needs as well as physicians' alike. Defying the problem, understanding the needs of the stakeholders and being aware of the gape that can bridge by technology are all together motivate this research work to build a solution. The main purpose is to develop a centralized effective system that helps to monitor heart disease patients remotely. The design artifacts include solution model and data analysis process. Creating these artifacts started with illustrating and then modelling the solution using suitable models. We demonstrated the design by Building the solution and the prototype which is consist from a) remote multi-sensor b) software application We then, evaluated the results as the following: a)a comparison of the outcome result with the objectives to discuss the contribution to the body of knowledge b) evaluating through conducting a survey with health specialists. We aim to communicate the solution to wider audiences, and other unaddressed issues related to these problems.

\section{THE FRAMEWORK}

Patients at home should wear a sensor and switch it on, the sensor starts capturing the vital signs data and buffer it into its repository. Whenever a Bluetooth connection is established with the PC, it starts downloading the data with the help of the SDK provided with the sensor. The downloaded data is a raw data, which includes information about different aspects of the sensor as well as the vital signs measurements. Patient need to be register into the system before they can benefit from it. So, this system has two sources of data. First one is coming from entering patient information manually throughout patient registration. Second source of data is the used sensor. Figure 1 shows an overview of the main parts of the system, and explains each part functions briefly. The data flow process is explained in details in the following section.

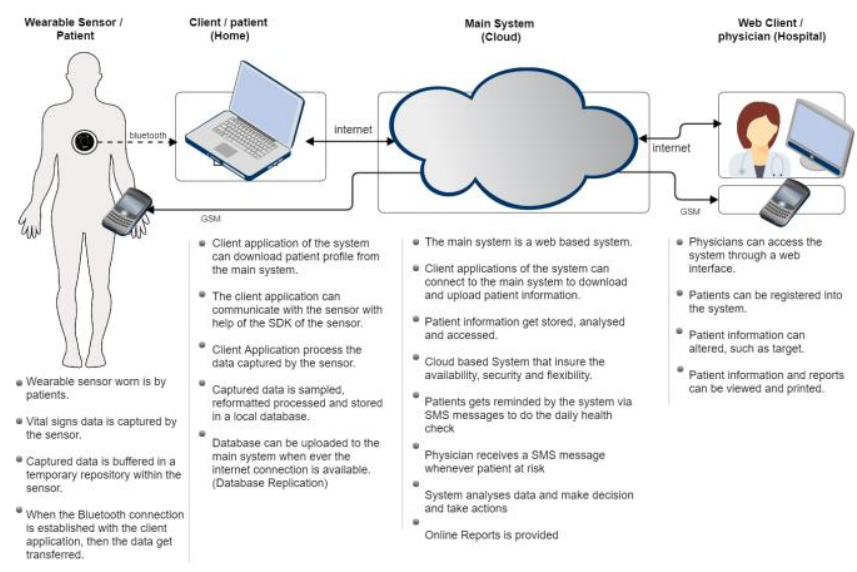

Fig. 1. Heart Disease Patient Remote Monitoring System Model

\section{A. Data Definitions}

Before diving deeply explaining how the system works, there are number of terms that used within this document need to be clarified, such as Vital Signs, Physical Activity, posture, Heartbeat Rate, Breath Rate and Heartbeat Rate variability.

Vital Signs: Vital Signs measurements is a routine procedure that takes place in any healthcare organization to monitor pulse rate (Heartbeat rate), breath rate, blood pressure and body temperature [17].

Physical Activity: Physical activity mode of the patient can be detected by the sensor. The sensor can determine whether the patient in a stationary mode, walking or in running mode. So, if activity level is less than 0.2 VMU then the patient is Inactive. If the rate is more than 0.2 VMU, then the patient is Active [18].

TABLE I. DETERMINING PATIENT PHYSICAL ACTIVITY

\begin{tabular}{|c|c|}
\hline Activity Value & Decision \\
\hline Activity value $<=0.2$ & Patient is inactive \\
\hline Activity value $>0.2$ & Patient is active \\
\hline
\end{tabular}


Posture: The sensor captures the body position of the patient. This will help identifying the state of the body while analyzing the heartbeat rate and breath rate. According to [18], position can be laying or standing, in which 0 degree means that the body is in a vertical position, 90 degree means that the body in a horizontal position, while 180 degree means that the body is inverted.

Heartbeat Rate (HR): According to [7], heartbeat rate means that the number of pulses occur in a minute. The normal rate range for children who is age between 6 and 15 is vary between 70 to 100 . Whereas, adults who are age above 18 are vary between 60 BPM and 100 BPM.

TABLE II. The Normal RATE OF HeARTBEAT RATE SEGMENTATION

\begin{tabular}{|c|c|}
\hline Age & Normal HR Rate (Beats Per Minutes BPM) \\
\hline Under age (6 - 15) & $70-100$ \\
\hline Adults (18 and over) & $60-100$ \\
\hline
\end{tabular}

Heartbeat rate can be calculated by calculating number of pulses during 10 seconds. Then multiply the number by 6 , that will provide the heartbeat rate. For instance, if a person blood pulse measured as 13 during ten seconds. Then $13 \times 6$ equals 78. So, the heartbeat rate for this person is 78 .

On the other hand, the maximum heart rate is important to be determined. The maximum rate is the highest level that can be achieved during a comprehensive exercise. To calculate the maximum heart rate, subtract the age from 220, then it will provide the maximum heart rate. For instance, a person whose age is 50 . Then by applying the equation (220 - 50) the result is 170 . So, the maximum heartbeat rate for this person is 170 beat per minute. Thus, physicians used to set a target for their patients and monitor their performance. The target starts from $50 \%$ to $85 \%$ of the maximum heartbeat rate. More than $85 \%$ is risky [7]. Table 3 shows how to calculate the maximum heartbeat rate as well as the calculation of different targets of the maximum heartbeat rate.

TABLE III. CALCULATION OF THE MAXIMUM HR AND THE AND THE TARGET OF THE MAXIMUM HR

\begin{tabular}{|c|c|c|c|c|}
\hline Age & $\begin{array}{c}\text { Maximum } \\
\text { HR } \\
(220-\text { age })\end{array}$ & $\begin{array}{c}\text { Target is } \\
\mathbf{6 0 \%} \\
(\text { Max. } \boldsymbol{H R} \boldsymbol{x} \\
\mathbf{0 . 6 0})\end{array}$ & $\begin{array}{c}\text { Target is } \\
\mathbf{7 0 \%} \\
(\text { Max. } \boldsymbol{H R} \boldsymbol{x} \\
\mathbf{0 . 7 0 )}\end{array}$ & $\begin{array}{c}\text { Target } \text { is } \\
\mathbf{8 5 \%} \\
(\text { Max } \boldsymbol{H R} \boldsymbol{x} \\
\mathbf{0 . 8 5})\end{array}$ \\
\hline $\mathbf{4 0}$ & 180 & 108 & 126 & 153 \\
\hline $\mathbf{5 0}$ & 170 & 102 & 119 & 144.5 \\
\hline $\mathbf{6 0}$ & 160 & 96 & 112 & 136 \\
\hline $\mathbf{8 0}$ & 140 & 84 & 98 & 119 \\
\hline
\end{tabular}

Breath Rate (BR): Breath Rate (BR) can be defined as the number of breathing per minute. Adult breathing normal rate is ranging from 12 to 20 time per minutes [7].

Heartbeat Rate Variability (HRV): "Heart rate variability, the change in the time intervals between adjacent heartbeats, is directly related to the body's interdependent regulatory systems and ultimately, their efficiency and health" [19].

\section{B. Data Gathering Process}

The process of data gathering and processing occurs through many steps. These steps start with patient registration, then vital signs data is gathered and the final step is data analysis.
These steps are going to be explained in further details in the following section. Figure 2 shows the whole process.

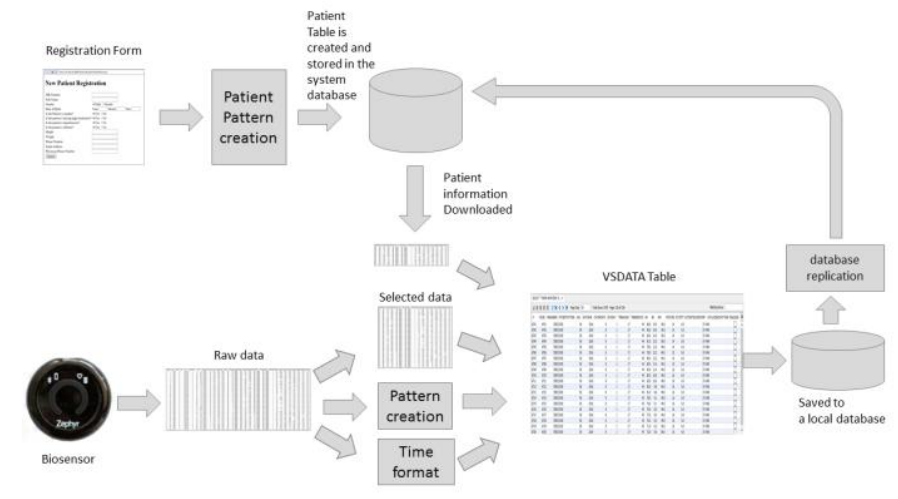

Fig. 2. System data flow model

\section{1) Patient Registration}

Patient registration is a step that should take place at hospitals or any healthcare facility by a person who have the authority to access the system. The following sections explain the steps that registration process goes through.

\section{a) Patient Information Entry}

The first step is to register patients in the system. Patient registration starts by filling a web form by an authorized person. The required information includes patient medical number, patient full name, gender, date of birth, heart rate maximum target, height, weight, patient telephone number, patient email and physician telephone number. Also, the collected information includes answers to four questions related to the patient's health problems and bad habits. The Health problem are diabetes, hypertension and cholesterol. While the bad habits that considered in patient registration is just smoking. Figure 3 shows a snapshot of the registration form.

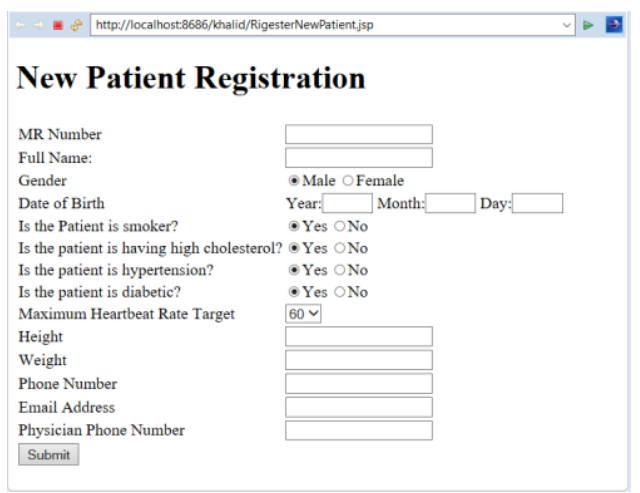

Fig. 3. Patient Registration Web Form

\section{b) Patient Profiling and Creation of Patient Pattern}

After filling up and submitting the registration from, and before it stored into the system database, the health problem and the bad habits information are processed in a way to create a pattern for the registered patient. Processing this part of data means that the system is creating pattern for this patient according to the answer of the provided questions. For instance, if the patient is smoker, then the value of this 
questions is capital " $\mathrm{S}$ ". while if he/ she is not smoker then the value is small " $\mathrm{s}$ ". This technique is applied to the health problems. Thus, $\mathrm{H}$ and $\mathrm{h}$ for hypertension, $\mathrm{C}$ and $\mathrm{c}$ for cholesterol and D and d for Diabetic. Eventually, the letters are combined according to the answers. Then the patient pattern code is created such as SchD, SCHD or schd. Figure 4 shows how the pattern is constructed.

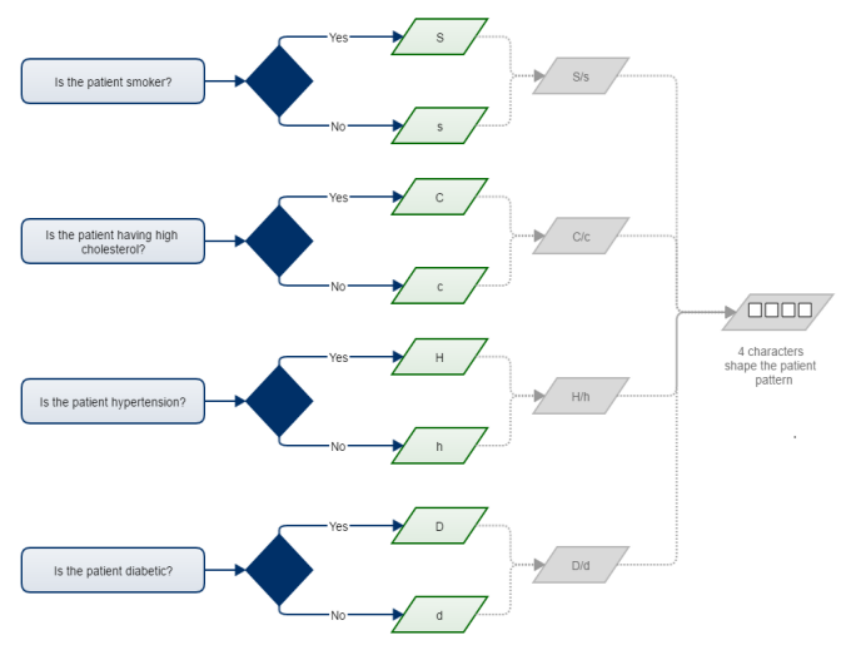

Fig. 4. The process of creating patient pattern

The aim of creating patient pattern is to classify patients and to set the target of maximum heart rate accordingly. So, physician can identify patients who are at risk, who are at less risk and who are out of the risk zone. Figure 5 shows all possible patterns that can be create out of this process. And it shows how the patterns are created. In addition, it shows patterns represent in different color code.

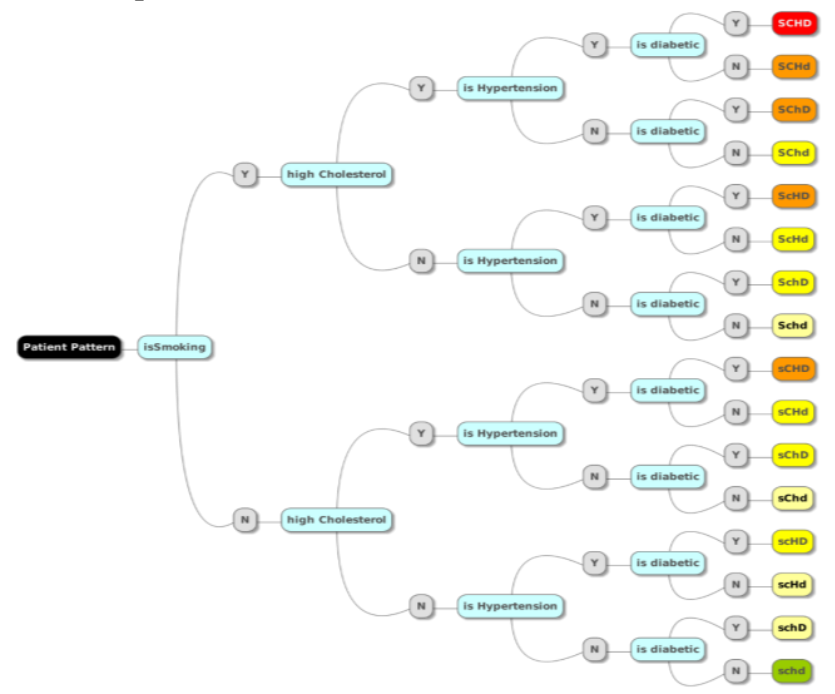

Fig. 5. All possible patient pattern with color code: Red is High risk, Orange is medium risk, Yellow is managed risk, Light Yellow is low risk and Green is no risk. a) Select Patient (Download Patient Information)

Patients at home start the client application. The application for the first time asks to sign in by providing patient record number. The application connects to the main system through the internet and retrieve the patient information. After that, the application is ready to process any captured data.

\section{b) Vital Signs Data Capturing (by the sensor - raw} data)

The client application (mentioned above) waiting for any new data to be added into a specified folder to read and process. Patients should wear the sensor properly first before they can switch it on. When the wearable sensor switched on, it starts reading the data and buffering the captured data in its repository. As soon as the Bluetooth connection is established between the sensor and the application, then the data starts downloading.

\section{c) Data Sampling, Data Formation and Vital Signs Pattern Creation}

Sampling the data step concerns with eliminating unwanted data fields and selecting the needed data from the raw data. After assembling the data, then the data is stored in a database. Before storing the data, some fields need to be refined and reformatted. For instance, time is given in milliseconds in the raw data. While the goal is make the time represented in hours and minutes. In order to utilize the time factor in later the data analysis step. In addition, this step involves creating new fields that hold a result of certain calculations of two or more fields. Pattern creation can be an example for that, and elaborate explanation will be given in this section. While we need specific data to satisfy the needs of this project. The sampled table stores data from two different sources of data; patient information and vital signs data.

\section{HeARtBeAt RATE DATA CAPTURING AND PROCESSING - CREATING THE FIRST PART OF THE PATTERN}

Full explanation has been given previously in this document regarding the heartbeat rate, the normal $\mathrm{HR}$, the maximum HR and the target HR. Despite this, it is worth it to repeat the part that related to how to calculate the maximum of HR and the target of maximum of HR. Because both of them going to be used in order to determine the vital signs pattern. MaxRate is a short form of Maximum Rate. Which can be calculated by this formula: 220 - age. While the Target is a short form of the Maximum Rate Target. It is a target that can be set by a physician to a patient. It starts from 50 percent till 85 of the MaxRate. For example, a patient with age 50 years old. Then, the MaxRate is 170 BPM (220-50). If the target is set as 60 percent, then the Target is equal to $102 \operatorname{BPM}(160 * 0.60)$.

The following table (Table 4) explains how the decision is made while building the vital signs pattern. it can be observed from the table that patient physical activity is 
considered. So, patients can be either active or inactive. The main reason of considering activity is that the HR normal rate of active patients differs from the normal rate of inactive patients. Also, it can be observed from the table that the posture involved before taking action if the HR below the normal rate. Because it is normal when the HR get down below the normal rate while the person is asleep.

TABLE IV. HOW THE DECISION IS MADE WHILE BUILDING THE VITAL SIGNS PATTERN IN RESPECT TO HR

\begin{tabular}{|c|c|c|c|}
\hline $\begin{array}{l}\text { Patient Physical } \\
\text { Activity State }\end{array}$ & $\begin{array}{c}\text { HR rate } \\
\text { value }\end{array}$ & Decision & $\begin{array}{c}\text { Denoted } \\
\text { by }\end{array}$ \\
\hline \multirow[t]{4}{*}{ Inactive } & $<60$ & $\begin{array}{l}\text { The HR rate is below the } \\
\text { normal }\end{array}$ & B \\
\hline & $60-100$ & $\begin{array}{c}\text { The HR rate is within the } \\
\text { normal rate }\end{array}$ & $\mathrm{N}$ \\
\hline & $\begin{array}{c}100 \text { - } \\
\text { MaxRate }\end{array}$ & $\begin{array}{c}\text { The HR rate is above the } \\
\text { normal rate }\end{array}$ & A \\
\hline & > MaxRate & $\begin{array}{c}\text { The HR rate is above the } \\
\text { maximum rate }\end{array}$ & M \\
\hline \multirow[t]{4}{*}{ Active } & $<60$ & $\begin{array}{c}\text { The HR rate is below the } \\
\text { normal }\end{array}$ & B \\
\hline & $60-$ Target & $\begin{array}{c}\text { The HR rate is within the } \\
\text { normal rate }\end{array}$ & $\mathrm{N}$ \\
\hline & $\begin{array}{l}\text { Target - } \\
\text { MaxRate }\end{array}$ & $\begin{array}{c}\text { The HR rate is above the } \\
\text { maximum rate }\end{array}$ & A \\
\hline & > MaxRate & $\begin{array}{c}\text { The HR rate is above the } \\
\text { maximum rate }\end{array}$ & M \\
\hline
\end{tabular}

\section{BREATHING RATE DATA CAPTURING AND PROCESSING - CREATING THE SECOND PART OF THE PATTERN}

Breathing rate value is captured from the raw data. According to the captured value, the decision is made and the pattern is created. And then, both the value and the pattern stored in the database. The following table (Table 5) explains how the decision is made.

TABLE V. HOW THE DECISION IS MADE WHILE BUILDING THE VITAL SIGNS PATTERN IN RESPECT TO BR

\begin{tabular}{|c|c|c|}
\hline BR value & Decision & Denoted by \\
\hline$<\mathbf{1 2}$ & The BR rate is below the normal & B \\
\hline $\mathbf{1 2} \boldsymbol{- 2 0}$ & The BR rate is within the normal rate & $\mathrm{N}$ \\
\hline$>\mathbf{2 0}$ & The BR rate is above the normal rate & A \\
\hline
\end{tabular}

At this stage, the system shall not take any action whatever the pattern is. That is a duty of alert analysis procedure, which takes necessary action for each pattern.

To sum up about vital signs pattern, creating vital signs pattern is one of the sub-processes that take place during the process of sampling data. Vital signs pattern is basically two English letters that represent two factors which are heartbeat rate and breathing rate. The first letter is representing heartbeat rate and the second is representing breath rate. So, the letter is changing according to the value of each factor. Both values are captured from the raw data. So, if the value of the factor is within the normal rate, then character denoted by "N". If the value below the normal rate, then the character denoted by "B". If value above the normal rate, then the character denoted by " $A$ ". If the value goes beyond the maximum rate, then the character denoted by "M".
Patterns are created for both heartbeat rate and breath rate to determine patient status.

Figure 6 shows a snapshot of VSDATA table, which is the main table in the system database. This snapshot shows how the pattern of vital signs is created in real. The first highlighted record shows that the value of the vital signs pattern is "NN". Which means that the heartbeat is normal and the breath rate is normal. While the second highlighted record shows that the pattern is "NB". The second letter (B) represent the breath rate. Which indicates that the breath rate of this patient is below the normal rate, and it can be observed that the value of $\mathrm{BR}$ is 10.6. which is below the normal rate which starts from 12 to 20. While calculating the HR and BR, the activity of the patient is considered. So, it obvious from the figure that the patient was inactive.

\begin{tabular}{|r|r|r|r|r|r|}
\hline \hline HR & BR & \multicolumn{1}{l}{ HRV } & POSTURE ACTIVTY & ESTIMATEDCORETEMP & VITALSCIENCEPATTERN \\
\hline 86.0 & 15.4 & 50.0 & 14 & 0.0 & $37.4 \mathrm{NN}$ \\
\hline 85.0 & 14.5 & 50.0 & 14 & 0.0 & $37.4 \mathrm{NN}$ \\
\hline 83.0 & 14.5 & 50.0 & 14 & 0.0 & $37.4 \mathrm{NN}$ \\
\hline 81.0 & 13.3 & 50.0 & 14 & 0.0 & $37.4 \mathrm{NN}$ \\
\hline 78.0 & 13.3 & 49.0 & 14 & 0.0 & $37.4 \mathrm{NN}$ \\
\hline 78.0 & 12.3 & 49.0 & 15 & 0.0 & $37.4 \mathrm{NN}$ \\
\hline 80.0 & 12.3 & 49.0 & 15 & 0.0 & $37.4 \mathrm{NN}$ \\
\hline 82.0 & 11.4 & 49.0 & 15 & 0.0 & $37.4 \mathrm{NB}$ \\
\hline 83.0 & 11.4 & 49.0 & 16 & 0.0 & $37.4 \mathrm{NB}$ \\
\hline 82.0 & 10.6 & 49.0 & 16 & 0.0 & $37.4 \mathrm{NB}$ \\
\hline 82.0 & 10.6 & 49.0 & 16 & 0.0 & $37.4 \mathrm{NB}$ \\
\hline 82.0 & 9.8 & 49.0 & 16 & 0.0 & $37.4 \mathrm{NB}$ \\
\hline 81.0 & 9.8 & 49.0 & 16 & 0.0 & $37.4 \mathrm{NB}$ \\
\hline 78.0 & 9.1 & 49.0 & 16 & 0.0 & $37.4 \mathrm{NB}$ \\
\hline 76.0 & 9.1 & 49.0 & 16 & 0.0 & $37.4 \mathrm{NB}$ \\
\hline 75.0 & 8.5 & 49.0 & 16 & 0.0 & $37.4 \mathrm{NB}$ \\
\hline 72.0 & 8.5 & 49.0 & 16 & 0.0 & $37.4 \mathrm{NB}$ \\
\hline 72.0 & 8.3 & 49.0 & 16 & 0.0 & $37.4 \mathrm{NB}$ \\
\hline 71.0 & 8.3 & 50.0 & 16 & 0.0 & $37.4 \mathrm{NB}$ \\
\hline 72.0 & 8.0 & 50.0 & 16 & 0.0 & \\
\hline
\end{tabular}

Fig. 6. Fragment from VSDATA table showing how the pattern of vital signs created in practice

\section{3) Data Analysis}

The system provides two types of data analysis; Alert Analysis and Patient Continuity Analysis. Both get executed by the system in a set time frame.

\section{a) Alert Analysis}

Alert analysis is kind of decision making process. Alert analysis focuses on the vital signs pattern. If the vital signs meet the alert criteria, then the caregiver or physician who looked after the patient will be notified with a SMS message with a short meaningful message. Figure 6 shows the decisions that made and the actions that taken for each pattern.

\section{b) Patient Continuity Analysis}

Patient Continuity Analysis is one of the main procedures of the system. This procedure consists of two sub-procedures. Both get executed automatically to follow up with patients to make sure they are doing the daily health check regularly. The health check means that patients should wear the sensor and run the client application of this system for a period of time (not less than 15 minutes). The first sub-procedure analyses patients' records, and send a SMS message to the patient who has no records. 
4)

\section{Reporting}

The system provides physicians with verity of reports that help them to get more clear picture about patients' health situations. These reports include Patient Risk Analysis Report, The Target of Heart Rate Maximum Rate Report, Daily Follow-up Report, Heartbeat Rate Analysis Report and Breath Rate Analysis Report.

\section{a) Patient Risk Analysis Report}

According to [20], Some bad habits and health problems considered the main factors that lead to heart attack or affect the health sate of hearts negatively. These factors include Smoking, cholesterol, blood pressure and diabetes. However, heart attack can be avoided by stop smoking and follow a diet that consider these health problems.

Patient Risk Analysis Report is a report that relying on patient pattern to assess the level of risk such as heart attack that patient might likely to face. So, as mentioned, patient patterns are 16 patterns, and each pattern associated with a risk level. On the other hand, the risk is divided into five levels; High Risk, Medium Risk, Managed Risk, Low Risk and No Risk. In addition, each level has a different color to help physician to differentiate between patients.

\section{The Technical Environment of The System}

The system consists of three main components: Sensor, Client Application and the Main System. These components will be explained in terms of their technical aspects, as well as their roles within the system.

Sensor: The employed sensor in this project is BioHarness 3.0 which developed by Zephyr Technology. This sensor consists of three components: chest strap, shoulder strap and the main module as shown in the Figure 7.

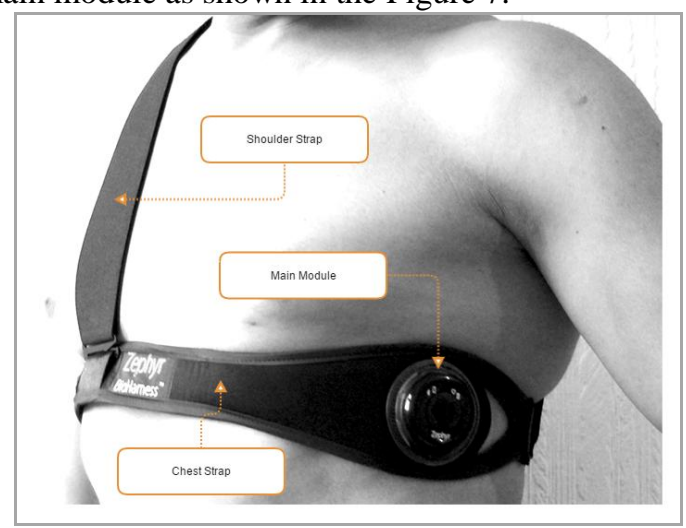

Fig. 7. The employed sensor (BioHarness 3.0) components

This sensor provides real-time monitoring capabilities as well as logging capabilities. Thus, Vital signs data such as heartbeat rate, breathing rate, body temperature as well as ECG and other readings are stored and transmitted by Bluetooth or USB cable. between the other captured data are activity state and patient posture. Activity sensor captures the accelerometer of the patient. So, it can determine whether the patient in a stationary mode, walking or in running mode. Posture sensor captures body position. The
SDK of the sensor comes with number of tools. First one is Cfg Tool. This tool is used to configure the sensor. So, many parameters can be set through this tool. The configuration can take place while the sensor is connected wirelessly through the Bluetooth or through a wired USB connection. Second tool is called Test App which can be used to view transmitted data. The role of the sensor is to capture the vital signs and ECG data and provide it the application in a format of CSV file. This sensor has decision making and alerting capabilities as well. But, only patients can benefit from this. Because the alerts are conveyed via LED lights on the face of the sensor itself.

Client Application: The client application is a windows desktop application developed based on Java platform, standard addition 8 development kit. Figure 8 shows the client application interface. It can be observed that the information of the patient with medical record number (5592) is downloaded and the application in a ready mode to collect and sample captured data.

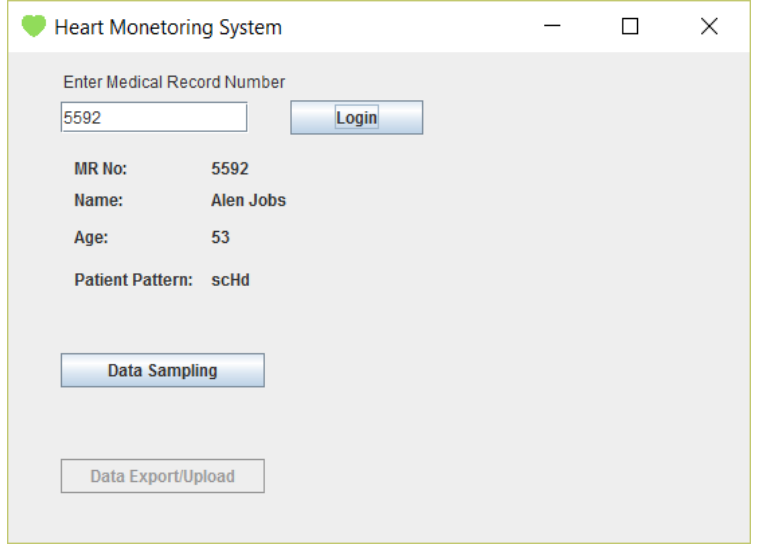

Fig. 8. The Client Application Interface

The role of the client application is to act as an intermediator between the sensor and the main system that hosted over cloud. It communicates with the sensor to capture the needed data and store it in a local database after analyzing it and prepare it to be uploaded to the database of the main system. The first step is to install the client application then to associate the application with the patient profile by providing the patient medical record number of the patient that intended to be monitored. The application provides three functions: Allow user to login to the main system, sampling and formatting captured data and upload the database to the main system.

Server System: The main system is a web based system. It is built based on Java technology with Web Standard Technologies, such as HTML, CSS and JavaScript. These web technologies further to JSP are used to represent data to users. And user can send a request While java code that executed Servlets classes are used to process and manipulate data. in addition, Servlet classes is used to manage the connection with the back-end database. Figure 9 shows the architecture of the main system. 


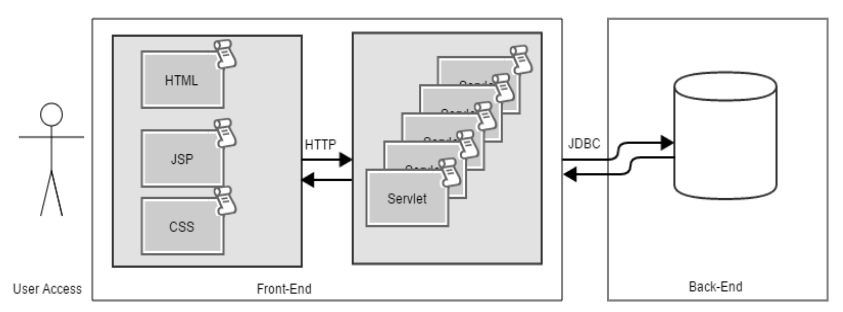

Fig. 9. Main System Architecture

The system has the services described in the table below:

\section{CONCLUSION AND FUTURE WORK}

Sensor technologies can be utilized effectively in healthcare systems. In particular, with chronic heart disease patients, as it helps to save lives by prompt intervention and reduce possible life loss to the half. Especially, if these technologies are utilized in patient remote monitoring systems. Thus, this paper introduced an effective remote monitoring system for heart disease patients by utilizing sensor technologies.

There are three stakeholder segments that can benefit from this research. First, this research can benefit heart disease patients. In which, the patient can stay at home and do the daily health checks without the need to go to the hospitals or any healthcare facilities. Second, it befits physicians and cardiologists. That means physicians might stop seeing their patient unless it is necessary. In other words, the system keeps on following up with the patient on a daily basis to reminding them to do the daily health check. And at the same time, the system alerts physicians about any abnormal results. So, this kind of automation might reduce the workload on physicians. finally, it benefits healthcare providers. In which, healthcare provider can integrate their healthcare systems with this system to provide a home care services.

For future work, we aim to provide a mobile version of the client application that will improve accessibility and ease of use. Other direction will focus on integrating the collected data with ECG data. We also aim at establishing a robust study on what wearable devices are more comfortable for patients and in which way it might help to improve their feelings about health. Another important idea that should be considered in future is utilizing data analytics mechanisms like cognitive analysis (e.g. Watson Analytics) which could potentially offer new perspective to the data analytics.

\section{REFERENCES}

[1] TechTarget, (2012). What is sensor? - Definition from WhatIs.com. [online] TechTarget. Available at: http://whatis.techtarget.com/definition/sensor [Last accessed 4 May 2018].

[2] T. Nick (2014). Did you know how many different kinds of sensors go inside a smartphone?. Phone Arena. [online] Available at: http://www.phonearena.com/news/Did-you-know-how-manydifferent-kinds-of-sensors-go-inside-a-smartphone_id57885 [LAst accessed 4 May. 2018].
[3] WHO, (2015). Cardiovascular diseases (CVDs). [online] World Health Organization. Available at: http://www.who.int/mediacentre/factsheets/fs317/en/ [LAst accessed 4 May 2018].

[4] J. Road, L. Oswego (2015). STUDY CLAIMS DEATHS DECREASE BY 50 $\%$ WITH REMOTE MONITORING. MedicalExpo [online] Available at: http://trends.medicalexpo.com/project-45815.html [Last accessed 4 May 2018].

[5] Faulx, M. (2014). Busting 5 Myths About Blood Pressure and Heart Rate Health Essentials from Cleveland Clinic. [online] Cleveland Clinic. Available at: https://health.clevelandclinic.org/2014/02/busting-5-myths-about-bloodpressure-and-heart-rate/ [Accessed 4 May 2018].

[6] AHA, (2016). Target Heart Rates. [online] American Heart Association. Available at: http://www.heart.org/HEARTORG/HealthyLiving/PhysicalActivity/FitnessBasi cs/Target-Heart-Rates_UCM_434341_Article.jsp [Accessed 24 May 2016].

[7] Cleveland Clinic, (2016). Hypertension (Normal vs. High Blood Pressure). [online]. Available http://my.clevelandclinic.org/health/diseases_conditions/hic_Hypertension_Hig h_Blood_Pressure [Accessed 24 May 2016].

[8] V. GAY, and P. LEIJDEKKERS, (2007). A Health Monitoring System Using Smart Phones and Wearable Sensors. International Journal of ARM, 8(2), pp.29-36.

[9] G. Appelboom, E. Camacho, M. Abraham, S. Bruce, E. Dumont, B. Zacharia, R. D'Amico, J. Slomian, J. Reginster, O. Bruyère, and E. Connolly, (2014). Smart wearable body sensors for patient self-assessment and monitoring. Arch Public Health, 72(1), p.28.

[10] A. Chan, N. Selvaraj, N. Ferdosi, and R. Narasimhan, (2013). Wireless patch sensor for remote monitoring of heart rate, respiration, activity, and falls. 2013 35th Annual International Conference of the IEEE Engineering in Medicine and Biology Society (EMBC).

[11] N. Selvaraj, (2014). Long-term remote monitoring of vital signs using a wireless patch sensor. IEEE Healthcare Innovation Conference (HIC), pp.83 86.

[12] A. Burrows, R. Gooberman-Hill, D. Coyle, (2015). Empirically derived user attributes for the design of home healthcare technologies. Personal and Ubiquitous Computing, 19(8), pp.1233-1245.

[13] R. Bloss, (2015) "Wearable sensors bring new benefits to continuous medical monitoring, real time physical activity assessment, baby monitoring and industrial applications", Sensor Review, Vol. 35 Iss: 2, pp.141 - 145

[14] L. Liu, E. Stroulia, I. Nikolaidis, A. Miguel-Cruz, and A. Rios Rincon, (2016). Smart homes and home health monitoring technologies for older adults. International Journal of Medical Informatics, 91, pp.44-59.

[15] N. Alshurafa, C. Sideris, M. Pourhomayoun, H. Kalantarian, M. Sarrafzadeh, and J. Eastwood, (2016). Remote Health Monitoring Outcome Success Prediction using Baseline and First Month Intervention Data. IEEE Journal of Biomedical and Health Informatics.

[16] K. Peffers, T. Tuunan T., M. A. Rothenberger, \& S. Chatterjee (2007). A design science research methodology for information systems research. Journal of management information systems, 24(3), 45-77.

[17] J. Hopkins. (2016). Vital Signs - Johns Hopkins Medicine Health Library. [online] Available

at: http://www.hopkinsmedicine.org/healthlibrary/conditions/cardiovascular_diseas es/vital_signs_body_temperature_pulse_rate_respiration_rate_blood_pressure_ 85,P00866/ [Last accessed 1 May. 2018].

[18] Zephyr Technology, (2016). Zephyr BioHarness 3 PRODUCT DESCRIPTION. 1 st ed. [ebook] Zephyr Technology. Available at: https://www.zephyranywhere.com/media/pdf/BH_DS_P-BioHarness3-DataSheet_20120919_V02.pdf [Accessed 2 May 2018].

[19] HeartMath. (2015). Article Explains Importance of Heart Rate Variability for Your Health - HeartMath Institute. [online] Available at: https://www.heartmath.org/articles-of-the-heart/science-of-the-heart/articleexplains-importance-of-heart-rate-variability-for-your-health/ [Last accessed 10 May. 2018].

[20] Emergency 101 - Heart Attack (2012) American College of Emergency Physicians. Avilable at: http://www.emergencycareforyou.org/emergency-101/ [Last accessed 10 May 2018]. 